\title{
Entre genre et santé : les espaces des transidentités
}

\section{Arnaud Alessandrin}

\section{OpenEdition \\ Journals}

Édition électronique

URL : http://journals.openedition.org/rfst/420

DOI : $10.4000 /$ rfst.420

ISSN : 2492-3672

Éditeur

Espaces et SOciétés (UMR 6590)

\section{Référence électronique}

Arnaud Alessandrin, «Entre genre et santé : les espaces des transidentités », Revue francophone sur la santé et les territoires [En ligne], Genre, territoire et santé, mis en ligne le 21 décembre 2015, consulté le 06 avril 2021. URL : http://journals.openedition.org/rfst/420 ; DOI : https://doi.org/10.4000/rfst.420

Ce document a été généré automatiquement le 6 avril 2021.

\section{(c) (i) (2) (2)}

La Revue francophone sur la santé et les territoires est mise à disposition selon les termes de la Licence Creative Commons Attribution - Pas d'Utilisation Commerciale - Partage dans les Mêmes Conditions 4.0 International. 


\title{
Entre genre et santé : les espaces des transidentités
}

\author{
Arnaud Alessandrin
}

\section{Introduction}

1 Les figures transidentitaires ont longtemps été contenues dans des espaces à la fois onscène (le cabaret, le monde de la nuit) et obscènes (la prostitution, les transformations corporelles). Psychiatrisation, pathologisation... le monde limitait ses espaces d'interactions avec les personnes trans à des sphères étouffées : le bloc opératoire et quelques journaux à scandale. Avec l'apparition d'un mouvement trans, structuré et politisé, la visibilité transidentitaire s'est accrue. La géographie des transidentités s'est elle-même épaissie, quittant le secret des cabinets médicaux et des biographies torturées, pour accéder aux interfaces écraniques, juridiques et donc publiques. Ainsi, les parcours trans questionnent la neutralité de l'espace et mettent en exergue sa dimension anxiogène, notamment lorsque l'expressivité de genre de la personne n'est pas conforme aux normes de genre en vigueur.

2 Pour cet article, je procéderai par étape - la géographie des transidentités posant ici ces premiers jalons. Il s'agira donc, dans un double mouvement, de revenir sur ce que la géographie a pu dire et écrire sur les transidentités, mais également de proposer des nouvelles pistes d'investigation. Dans un premier temps, je tenterais un état des lieux de la littérature qui conjugue l'espace et les transidentités. Puis nous examinerons la manière dont le territoire, la santé et les transitions trans se mêlent dans des espaces plus ou moins anxiogènes, plus ou moins visibles aussi, et la manière dont l'écume des mouvements féministes et homosexuels balisent un espace public tout à la fois interface d'expressivités dissidentes et opérateur des normes de genre. A travers quelques témoignages, je reviendrais sur les lieux de la transition (les espaces médicaux et juridiques) avant d'interroger les lieux du quotidien ainsi que les espaces et les temporalités créées par les trans, pour les trans. Méthodologiquement, cet article prend appui sur trois qualitatifs et quantitatifs : une thèse en sociologie portant sur les 
transidentités (Alessandrin, 2012), puis sur une recherche sur la transphobie (Alessandrin et Espineira, 2015), et enfin sur une recherche plus générale sur les discriminations, qui inclue des personnes trans ${ }^{1}$

\section{L'espace des transidentités : état des lieux}

\section{Transidentités : de quoi parle-t-on?}

3 Parler de transidentité, nécessite que l'on revienne même brièvement sur les termes employés et sur l'histoire des concepts. Le "transsexualisme " est une invention psychiatrique datant de 1952. La prise en charge de ce "transsexualisme» est, en France tout du moins, assez récente (Alessandrin, 2012). Dès les années quatre-vingt, des protocoles hospitaliers sont mis en place. Actuellement au nombre de cinq (deux sur Paris, un à Bordeaux, Lyon, Marseille), ces derniers instaurent des procédures de sélections à l'entrée et durant les parcours de soins. S'ils ne sont en rien obligatoires pour vivre une transidentité, ces protocoles psychiatriques assurent un remboursement de l'opération par la sécurité sociale et permettent l'obtention d'un changement d'état civil facilité à la suite d'une opération de réassignation, parfois vécue comme une injonction à la stérilisation. En effet, c'est à la condition d'une opération irréversible, trop souvent entendue comme une stérilisation, et d'un suivi psychiatrique, que les tribunaux français accordent, sur la base de jurisprudences éparses et contradictoires, des changements d'état civil aux personnes requérantes. Dans ce contexte, les parcours de transition sont variés et, comme l'aura montré l'enquête de l'INSERM (2011), de nombreuses personnes trans préfèrent ne pas se faire en France ni même bénéficier d'une opération de réassignation. Ces parcours multiples cachent aussi des réalités complexes, marquées par le sceau de la transphobie. L'enquête menée par Arnaud Alessandrin et Karine Espineira en 2014 montre par exemple que $85 \%$ des trans ont subi un acte transphobe durant les douze derniers mois. Pour plus de 37\% d'entre eux, cet acte s'est reproduit plus de cinq fois. Pour plus de la moitié des enquêté.e.s (58\%), l'état civil est la première cause de discrimination. Si l'on se penche sur les formes de la transphobie, on observe que les insultes et les discriminations reviennent dans $60 \%$ des cas. Si Internet est montré du doigt comme espace de discriminations ( $30 \%$ des réponses), la rue (plus de $50 \%$ ), le travail et la famille (plus de 30\%) sont également mis en lumière. La transphobie est un fait de l'espace public. Quant au monde médical, il a été le théâtre de discriminations pour les $2 / 3$ des répondant.e.s! Les écueils dans une transition sont donc potentiellement partout. Dans la sphère publique comme dans les espaces les plus intimes. Mais au total, seuls $3 \%$ des trans victimes de discrimination portent plainte... Là encore par peur que le secourant soit lui-même transphobe. La question des espaces de la transphobie est donc immédiatement corrélée à celle des transidentités.

\section{Géographie des transidentités.fr}

$4 \quad$ En France, les recherches autour des espaces des transidentités sont assez limitées. Les trans studies elles-mêmes nouvelles dans le champ académique français (Espineira, 2008) et peinent parfois à se faire entendre (Espineira, 2013). À ce titre, les tentatives de géographies LGBT restent majoritairement des analyses « gays » (Jaurand, 2005 ; Leroy, 2005, 2009 ; Raibaud, 2013) ou « lesbiennes» (Clerval et Cattan, 2011; Amari, 2013). On 
pourra noter, parmi quelques auteurs cités, non pas une mise à l'écart de la question transidentitaire, mais plus spécifiquement une volonté déontologique de ne pas traiter d'une question éloignée de leurs terrains d'enquêtes. Ainsi, Marianne Blidon précise-telle, dans sa conclusion du livre "Géographie des homophobies" (2013) que son " propos s'attachera aux gays et aux lesbiennes. Certains points peuvent s'appliquer aux transgenres et aux transsexuels. Je n'ai cependant pas souhaité faire l'amalgame entre homophobie et transphobie et traiter la question trans comme un corolaire de la question gay et lesbienne. La transphobie relève d'une logique propre à la transgression des normes de genre et non des normes sexuelles. A ce titre, elle mérite d'être prise au sérieux et de faire l'objet d'une étude spécifiques afin d'y apporter les réponses les plus adaptées »(p.269). C'est aussi le cas de Nadine Cattan et Stéphane Leroy qui, dans leur «Atlas des sexualités» (2013), insistent sur le fait que " les données sérieuses pour aborder la sexualité des transsexuel.le.s par exemple sont indisponibles tant la sexualité de ces groupes de personne est encore tabou dans les sociétés contemporaines » (p.92). Les trans studies ne sont donc pas encore entrées dans la géographie française. Quelques textes, issus du livre "Géographie des homophobies » font néanmoins exception. Celui de Maud Yeuse Thomas tout d'abord, prenant appui l'exemple des « UEEH ». Les Université d'Eté Euro-méditerranéenne des Homosexualités (Thomas, 2012) proposent tous les ans un "espace» qui est aussi un " temps » d'échanges et de découvertes dans lequel les multiples expressivités de genre et de sexualité sont négociées entre tou.te.s les participant.e.s. Ici, l'espace est un espace "queer" au sens où les identités et les corps créent des espaces qui sont mouvants, poreux et consentis (la plus part du temps) aussi bien en mixité qu'en nonmixité pour les filles, les trans ou toute autre auto-appellation (Thomas, 2013). Le lieu de ces rencontres, l'université de Luminy à Marseille, redevenant, immédiatement après le festival, ce qu'il était avant l'évènement: un espace étudiant et artistique. Entre expériences hyperlocales et mouvement global, la question trans se définit aussi par des espaces interstitiels, dont la territorialisation est tissée de représentations, de narrations et de constructions matérielles. C'est par exemple le cas des espaces de la prostitution comme le «bois de Boulogne» dont on pourra dire aux côtés de Karine Espineira qu'ils ont littéralement été créés par un discours médiatique ; lequel discours venant à couper un espace intégré, sain, d'un espace montré comme obscène (et paradoxalement mis sur la scène médiatique), celui des prostitué.e.s et des travestis, avec pour toile de fond la question de la migration (Espineira, 2013 ; 2014). Néanmoins, dans ces travaux, ce n'est pas à proprement parler le territoire qui est appréhendé en premier lieu. D'ailleurs, ces cheurcheuses et chercheuses ne sont eux-mêmes pas géographes. Toutefois, si cela démontre uns possibilité de dialogue entre trans studies et géographie, et s'il convient de noter l'émergence d'une géographie queer en France (Borghi 2012, 2014; Blidon, 2006), cela ne permet pas de conclure que la géographie française a intégré la question trans Peut-être, faut-il alors se pencher du côté des productions de jeunes chercheurs, pour trouver des prémisses à une géographie des transidentités, par des géographes notamment. A ce titre, le mémoire de Yoan Gutowski (sous la direction de Claire Hancock, 2013) et intitulé « La transphobie et son influence sur les pratiques des personnes transidentitaires dans l'espace public ", aura tenté, dans une oscillation entre sociologie et géographie des transidentités, d'illustrer quelques difficultés ressenties par les personnes trans dans leurs déplacements dans l'espace, y compris dans des espaces dits "communautaires" comme le marais ${ }^{2}$. La rareté de ces travaux est d'autant plus étonnante que le terrain transidentitaire se fait écho d'un grand nombre d'écueil dans la quotidienneté des déplacements et des 
interactions spatiales. L'association OUTrans (2013) exprime à cet égard les tentatives de prises d'espaces par les trans lors de manifestations comme « Existrans ", mais aussi lors de rassemblements thématiques (sur le VIH ou la prostitution par exemple), allant à l'encontre d'une certaine invisibilité, parfois souhaitée par conformité ou sécurité et d'autre fois subie par absence de connaissances partagées sur les question trans au-delà d'un cercle restreints de militant.e.s et de personnes concernées. Enfin, la géographie des transidentités s'élargit si l'on considère l'ensemble des transgressions à l'ordre de genre. Les évènements comme la marche des fiertés (Leroy, 2010) donnent aussi à voir un «dépassement d'une ligne invisible» (Lestrade, 2003) ne concernant pas que les questions sexuelles. De même, une géographie des figures folles (Alessandrin, 2014 ; Le Talec, 2013) ou des mouvements intersexes (Bastie-Charlebois et Guillot, 2013) viennent éclairer la manière dont les questions de santé et les questions de genre dessinent des territoires paradoxaux, tout à la fois visibles et médiatiques, aussi bien qu'inaudibles et marginaux.

\section{Géographie des transidentités.com}

5 C'est en se penchant sur les productions internationales, que la moisson devient un peu plus abondante. Dans le Transgender studies reader de Susan Stryker et Stephen Whittle (2006), Viviane Namaste revient sur le genderbashing (Namaste, 1996) dont sont victimes les personnes trans (p.584) et Anne Wilchins souligne les risques et les stratégies du coming out trans dans un article intitulé « What does it cost to tell the truth?» (p.547). On soulignera de même la présence d'un questionnement autour des figures trans dans l'espace public autour de quelques textes inauguraux de la queer théory , comme «In a queer time and place » de Judith Halberstam (2005) ou bien « Le mouvement transgenre» de Pat Califia (2003). La visibilité trans prend donc simultanément appui sur des territoires à la fois physiques et médiatiques. Il convient de noter que cette dernière dimension prend souvent le pas sur la première. C'est par exemple le cas dans le livre dirigé par Chantal Zabus « Transgender experience : place, ethnicity and visibility ( 2014) qui entend par "visibilité », les interfaces publiques, politique ou culturelles qui font que s'il n'existe pas de «culture trans » il existe plus précisément des figures trans dans la culture (Alessandrin, 2012). De ce point de vue, les récits de vie ou les blogs trans, interrogent la place de l'autobiographie et des expériences singulières dans la restitution d'une géographie des transidentités (Moss, 2001). Les publications de Petra Doan ${ }^{3}$ sont ici d'une importance capitale puisqu'elles semblent les seules à traiter, géographiquement, des expériences spatiales et sociales de transidentité. Deux publications dans la revue «Gender, Place \& Culture : a Journal of Feminist Geography" retiennent alors mon attention. La première, intitulée "Queers in the American City: transgendered perceptions of urban space» (Doan, 2007) revient, à partir de 149 témoignages, sur l'injonction sociale et spatiale à la binarité de genre et sur les violences à l'encontre des individus qui la transgressent. La question de l'espace public comme lieu potentiellement dangereux a déjà été reprise de nombreuses fois dans les études sur les transidentités et sur la transphobie (Hill, 2002), mais sans forcément que celle-ci soit spécifiquement en lien avec une coloration géographique. Le second article, intitulé « The tyranny of gendered spaces - reflections from beyond the gender dichotomy" (Doan, 2010) propose une écriture "autoethnographique" que l'auteur explique de la sorte: "Dans les pages suivantes j'écrirais autoethnographiquement ce qui renvoie à mes propres expériences genrées de l'espace, 
du point de vue d'une femme transgenre " (p.636). Dans la suite des travaux en autoethnographie (Ellis, 2004), ces deux articles soulignent les rares interstices de sécurité et de tranquillités laissés aux personnes trans (et intersexes) et sont écrits, comme il est plus rare de le voir en France, de par l'expérience des personnes concernées. On notera de ce point de vue, comme cela a pu être le cas des études homosexuelles ou féministes, que les frontières entre les savoirs expérientiels et savants, profanes et universitaires, sont plus poreuses qu'il n'y parait. Enfin, il convient de souligner le travail géographique Katherine Browne. Un premier article interroge la place des trans dans les quartiers gays de Londres (Browne, 2010). L'auteure y rappelle les tensions inhérentes aux espoirs d'inclusion dans la communauté LGBT et, simultanément, les expériences de marginalisation auxquels les trans font face dans cette même communauté. Dans le même numéro de revue, la recherche propose d'intégrer les questions trans aux questionnements géographiques. Si son appel n'a pour l'instant pas trouvé un écho réel, il souligne bien la place laissée vacante face à ces questions.

6 Il y a donc beaucoup à faire si l'on veut faire se rejoindre les préoccupations sociales de la géographie du genre et les expériences transidentitaires, dans leurs diversités. Des enquêtes plus ciblées pourraient ainsi nous aider à saisir ce qui se joue à l'intersection de ces deux champs. Par exemple, une enquête commandée par la ville de Liverpool et réalisée par Catherine Reynolds sur la vie des personnes trans (2011), met en lumière les problèmes de voisinages, de déplacement des personnes trans, y compris dans les transports en commun ${ }^{4}$. Steve, un jeune trans, évoque sous ces termes les difficultés à prendre le bus : « Je me bats pour prendre le bus. Je devais prendre le bus pour aller au collège, mais je viens juste d'acheter une bicyclette car je ne parviens plus à le supporter. Je ne sais pas si c'est moi mais je sens que tout le monde me regarde, m'analyse " (p.37). C'est dire combien la géographie a toute sa place lorsqu'il s'agit d'envisager l'espace comme un espace de vie vivable pour les uns, un espace anxiogène pour les autres, sur quoi nous pouvons nous appuyer pour vivre une vie humaine à côtés d'autres vies humaines, en considérant par là même que lorsque l'espace public n'est plus accessible à tous, il s'estompe au profit de « murs invisibles » (Di Meo, 2012) segmentant les expériences et les existences.

\section{Borders that matters}

7 Karine Espineira (2008) comme Maxime Foerster (2008) ont souligné bien avant moi, le passage d'un espace clos, psychiatrisé, à un espace public sous tensions ; c'est-à-dire aussi à des visibilités complexes, parfois fragiles. Ils ont aussi mis en avant l'existence des manifestations et d'expressivités trans plus ou moins impactantes ${ }^{5}$ : des associations locales; des rassemblements nationaux; des actions d'auto-support; des actions juridiques menées auprès de la cour européenne de justice. Les échelles de l'action militante trans, et donc des visibilités trans, sont multiples et augmentent ainsi les interfaces de conflit. De ce point de vue, l'histoire des transidentités est révélatrice des écarts aux normes de genre qui vont être tus, prohibés ou psychiatrisés, et pour lesquels on va préférer déployer des technologies qui changent le corps plus que les normes (Alessandrin, 2012b). L'analyse des territoires trans, à la jonction entre les parcours de santé et les parcours de genre des personnes trans, peut alors prendre appui sur les espaces mis en exergue dans les récits de vie mais aussi sur les espaces mis en scènes dans les médias afin d'en souligner l'importance analytique. Nous travaillerons ici sur les lieux du quotidien (l'école, la famille) ainsi sur les lieux de la 
transition médicale (en se limitant aux protocoles hospitaliers) pour mettre en évidence les multiples théâtres de la transphobie. Pour cette partie, il convient enfin d'entendre la notion de santé au sens du bien-être et la notion d'espace, parfois métaphorisée, au sens de la scène dans laquelle se déroule l'action.

\section{Géographie de l'intime}

8 Les témoignages trans insistent fréquemment sur la dimension à la fois rare et précieuse des espaces intimes, soumis à de multiples contrôles, aussi bien des parents, des proches ou des médecins, venant faire du privé un espace meurtri, traqué, psychiatrisé. A la suite des travaux en géographie de l'intime et des émotions ${ }^{6}$ (Bochet, 2002), l'espace scolaire et les pièces privatisables dans la maison sont d'excellents exemples afin de rendre compte des tensions existantes entre la police du genre et l'expression d'une identité de genre dissidente.

9 Premiers exemples de cette gestion de l'intime dans l'espace: les lieux scolaires qui traduisent bien ces instants anxiogènes de la cour, de la classe, des toilettes ou de la cantine. Des lieux collectifs, marqués par le sceau du masculin (Ayral, 2011) qui font de l'espace de l'école, du collège ou du lycée, des espaces genrés, marqués par la contrainte. Camille a 32ans. Au souvenir de ses années de collège elle partage une expérience douloureuse : Pour beaucoup de trans, aller aux toilettes des garçons (ça marche aussi pour les filles) ça a juste été un cauchemar. Moi j'ai longtemps évité les toilettes. La cantine aussi d'ailleurs. Je me retenais. Le pire je crois c'est les cours de sport. Se déshabiller, être viril et tout. C'était terrible. Je te dis pas le nombre d'absences que j'ai dû justifier! (Camille) . (Alessandrin, 2014b)

10 Parce qu'ils n'ont pas les mots ou parce que ces derniers sont irrecevables, les jeunes trans restent par exemple souvent dans l'ombre d'une identité malmenée : « Dès le plus jeune âge je savais que j'étais une fille. Mais je l'ai refoulé. Et puis je ne savais pas ce que c'était. Au début j'ai cru que j'étais un garçon homosexuel. J'ai mis du temps à m'assumer. En fait, j'ai surtout mis du temps à savoir qu'on pouvait être transsexuelle " (Marie, 52 ans). Dans le processus qui mène à se nier, les personnes trans déploient des grammaires par défaut. Il s'agit aussi « de ne pas paraître » ou « de ne pas être » celui qu'on perçoit ou celui qui serait susceptible d'être démasqué : J'ai à peu près tout fait comme il fallait. Pour qu'on me laisse tranquille mais surtout parce que je me trouvais anormale. J'ai fait "sciences", puis une école d'" ingé ". J'ai aussi fait l'armée. Un mec quoi, un vrai. Mais plus j'étais ensevelie sous des couches, plus je me sentais loin de qui j'étais vraiment " (Laetitia, 44 ans). Contrairement à l'homosexualité, et malgré Internet, la connaissance du monde trans et de sa propre transidentité est un chemin semé d'embûches. Une second différence se fait jour sur la question de l' outing ou de la nécessité du coming out trans. Dans le contexte actuel du droit, et pour les personnes trans qui n'ont pas pu bénéficier d'un changement d'état civil, chaque sortie des papiers d'identité pose la question du coming out et de l' outing : " J'étais à la caisse d'un super marché et j'ai voulu payer avec mon chéquier. Lorsque j'ai sorti ma carte d'identité, la caissière a refusé de prendre mon chèque. J'ai donc dû lui expliquer la situation. Les gens autour de moi nous regardaient avec étonnement, ils n'avaient surement jamais entendu ça "(Christian, 60 ans). Un autre témoignage rappelle les outing fréquents et mal maitrisables: "A pôle emploi, j'avais rendez-vous avec une conseillère. Comme elles changent tout le temps je dois toujours m'expliquer sur ma situation. Et la dernière fois, j'étais assise en train d'attendre quand j'entends mon nom : «Monsieur X». Qu'est-ce que tu 
voulais que je fasse? J'ai pris sur moi, je me suis levé, j'ai été suivie du regard par l'ensemble des personnes présentes et j'ai dû expliquer de nouveau ma situation... " (Emmanuelle, 51 ans). Un autre lieu attire l'attention lors d'une analyse qui croise la santé et les territoires trans : celui de l'habitat et plus précisément de la chambre et de la salle de bain. Julie a 55ans et a deux enfants. Lorsqu'elle était mariée, la maison s'organisait autour des rôles de genre que la famille attribuait à chacun des membres. " En tant que père de famille, ils ne pouvaient pas me voir autrement que comme un homme. J'ai dû planquer mes robes, mon maquillage et tout. C'est pas que j'avais honte mais je ne voulais pas qu'ils le voient. Et puis je me cherchais. » Pour vivre son identité de genre Julie a acheté un camping-car. " C'était super parce qu'on pouvait partir en vacance tous ensemble et moi je pouvais aller en ville et me changer dans mon camping-car sans que personne ne me voit et sans que je me fasse agresser du fait que je n'avais jamais le temps dans la salle de bain ou dans la chambre pour me changer, bien me maquiller tout ça... ». Les temporalités du couple ou de la sphère familiale sont ici convoquées dans une tension entre peur et réconfort.

\section{L'espace du diagnostic est-il un espace de santé ?}

11 Le témoignage de Julie s'engage également du côté de la santé. "Quand la psychiatre a voulu que je fasse mon real life test ${ }^{7}$, je me suis dit 'tu peux pas venir habillée en homme, il faut que tu montres qui tu es'. Sur le chemin, je m'arrêtais au local LGBT: comme ça je partais de chez moi en homme et j'arrivais chez elle en femme. En fait, la psychiatre il faut la convaincre. Mais il faut aussi faire attention à la discrimination, parce que quand tu prends le bus pour la première fois comme ça et bien tu peux te faire embêter parce que tu n'as pas un bon passing "

12 Le "passing " signifie l'action de passer, passer en direction d'un genre, mais aussi passer « pour ». Dans l'incertitude du passing, logées dans l'épaisseur des interactions quotidiennes, les personnes trans subissent une double injonction à l'assimilation aux normes de genre. Une injonction sociale d'abord puisqu'il s'agit, dans le but d'amoindrir le coût des interactions potentiellement dangereuses, de maximiser l'adéquation aux normes de genre. Une injonction psychiatrique ensuite, puisque la réussite du passing, est une condition à la poursuite des protocoles hospitaliers de changement de sexe. Ainsi, du côté des patients trans, l'espace de la santé est soumis à des peurs, à des craintes. C'est ce que l'enquête de l'association Chysalide met en lumière. Selon cette enquête, $16 \%$ des trans se sont vus refuser des soins du fait de leur transidentité en 2010 et que $35 \%$ des trans ont renoncé à des soins du fait de préjugés ou de discriminations émanant du personnel de santé. $57 \%$ des sondés estiment enfin avoir été gênés par l'attitude des soignants ${ }^{8}$. S'ajoutent à cela les craintes relatives au passing, c'est-à-dire, par exemple, à l'accueil médical et aux déplacements jusqu'au rendez-vous, qui sont autant d'obstacle anticipés qui nuisent à l'accès aux soins. Audrey a 37ans. Elle appréhende toujours le rendez-vous dans l'hôpital public qui la suit. Elle sait qu'en fonction des infirmièr.e.s ou du personnel d'accueil, elle sera plus ou moins bien genrée. "Quand il y a des « monsieur X » qui sont lancés devant plein de monde à l'hôpital et que tu dois te lever pour aller chercher ta feuille ou suivre l'infirmière, t'as tellement honte, surtout au début, t'as tellement honte que t'as pas du tout envie de faire un scandale ou de dire "non c'est madame ». Je leur ai répété des centaines de fois mais c'est comme s'ils n'écoutaient pas ». Plus souvent, les témoignages mettent l'accent sur les praticiens mêmes des protocoles. Des situations de vives tensions apparaissent. Laurent est un jeune garçon trans venu dans une ville qui héberge un protocole afin de bénéficier d'un suivi. Après deux ans de rendez-vous chez la 
psychiatre de ce protocole, on lui annonce qu'il ne pourra pas continuer avec cette équipe : « je pensais qu'on m'annoncerait la date de l'opération et en fait ils m'ont juste dit que je n'étais pas transsexuel, car je ne voulais pas de phalloplastie (construction d'un pénis). J'ai tenté de leur dire que j'avais déménagé, que j'avais quitté mon travail pour pouvoir faire cette opération ici mais ils n'ont pas voulu entendre. » L'espace du soin devient alors un espace de méfiance. "Tout ce que tu dis, tout ce que tu fais c'est retenu contre toi: t'es homo, t'es séropo, tu te prostitues, t'as eu des enfants, t'es pauvre ou je ne sais pas quoi, à chaque fois y'a une bonne raison pour qu'ils te virent ou pour qu'ils repoussent la date des opérations » note Christian, 62ans, dont l'opération a également été repoussée pour respecter les trois ans de suivis psychiatriques préalables à l'opération et demandés par le chirurgien. Paradoxalement, les protocoles de changement de sexe éloignent donc les personnes trans des espaces de santé qui leur sont consacrés.

\section{Médias et espaces trans}

13 Avant Internet et l'instauration des protocoles tels que nous les connaissons (Alessandrin, 2012), les individus se payaient eux-mêmes leurs opérations. La géographie du changement de sexe était donc déjà une géographie des marchés de santé dans laquelle le silence côtoyait la peur. Dans le reportage " Nous n'irons plus au bois $"{ }^{9}$, Josée Dayan montre les réseaux et les combines pour financer les opérations et les voyages, parmi lesquelles la prostitution ou le cabaret. Entre invisibilité et interventions policières, les lieux trans demeurent, certes actifs, mais précaires.

«- Revenons-en à la police... Qu'en pensez-vous?

- Ils sont odieux; ils vous emmènent comme ça sans raison... et quand ils nous arrêtent ils nous parlent au masculin... chaque fois qu'on est «emballée " on attrape 150 Francs d'amende pour racolage : 130 Francs - ce qui est le tarif normal plus 20 Francs compte tenu du fait qu'on est travesti » (Piat, 1979)

On retrouve les mêmes témoignages dans un autre reportage, américain celui-ci, réalisé par l'activiste et historienne trans Susan Stryker. Intitulé " Screaming queens » ${ }^{10}$, ce dernier rend visibles des vies géographiquement et professionnellement ostracisées. Tout se déroule entre quelques ruelles d'un quartier de San Francisco et autour d'un bar (le "Compton's cafeteria ») dans lequel les «queens» sont fréquemment arrêtées par la police. La "transsexualité », comme trouble et comme solution, se diffuse rapidement grâce notamment à une forte médiatisation.

Si, dans les médias traitant de la transidentité, le vocabulaire de la souffrance (et donc du courage et de la sollicitude) est saillant aujourd'hui, à l'époque de Christine Jorgensen ou de Coccinelle (premières figures trans médiatisées) on oscille entre réprobation morale et voyeurisme étonné. Dans son essai sur les transidentités dans les médias, Karine Espineira relève un extrait télévisuel de 1962 dans lequel la visibilité de Coccinelle est soumise à la suspicion du « faux genre ».

«Encore un détour par la plage. Qui donc regarde cette foule manifestement inspirée? Une femme naturellement. Quelle question ?! Et naturellement, de la meilleure espèce. Il n'y a qu'un ennui. Cette femme est en effet un homme ! Il paraît même qu'elle s'est mariée à l'Église. Qui donc pourrait songer à mal? Pas elle. Ou lui. Comme il vous plaira. Mais cela vous plait-il tellement? Pas à nous!» (Espineira, 2008, p.17) 
16 La géographie des transidentités est donc une combinaison de représentations onscènes aux yeux du public et obscènes aux yeux de la société et du droit. Plus récemment, ce sont des films comme « Transamérica » ${ }^{11}$ ou « Laurence anyways " ${ }^{12}$ qui nous ont permis d'appréhender la manière dont les personnes trans bricolaient des espaces dans des lieux parfois hostiles. Ainsi, dans Transamerica, Bree se voit conseiller par une amie, pour plus de sécurité, de se faire héberger par un groupe de personnes trans au cours de son voyage. Dans le même mouvement, dans Laurence anyways , Laurence est recueillie après une agression par une famille d'artistes vivant derrière un rideau de scène. Dans ces deux films, la visibilité et son coût dans l'espace public esquissent des lieux, des espaces dans l'espace, qui, tout à la fois, protègent et ostracisent de nouveau.

\section{Conclusion : l'espaces cisgenre}

17 Pour terminer, il faudra souligner que la géographie des transidentités prend appui sur les acquis des luttes homosexuelles et féministes. Elle rebat les cartes favorables à une lecture neutre ou impersonnelle de l'espace et inaugure une lecture non-cisgenre (Serano, 2007; Alessandrin, 2014c), c'est-à-dire qu'elle interroge l'évidence d'une adéquation sexe-genre dans les interactions publiques et dans la présentation de soi. Une géographie des transidentités se révèle donc, en creux, être une géographie du « privilège cisgenre » pour reprendre l'expression de Julia Serano, même si, les notions de cis-identité (dans une réitération de la bipartition de genre) et de privilège (qui s'oppose à une définition relationnelle du pouvoir), pourraient elles-mêmes être relues à l'aune de réalités plus intersectionnelles. En effet, les derniers travaux sur la transphobie (Alessandrin et Espineira, 2015) montrent bien l'importance qu'il y a à saisir les entrecroisements catégoriels qui favorisent telle ou telle expression de la discrimination. Ainsi, il conviendrait de revenir plus précisément sur l'expérience spatiale des femmes et des hommes trans mais également sur leurs expériences comparées en termes d'âge ou de racialisation. Les transidentités sont plurielles ainsi qu'il faut envisager une géographie des transidentités, ainsi qu'une géographie des formes de transphobie. De ce point de vue, la question trans permet de revisiter des problématiques liées à l'approche de la santé par les territoires. Elle insiste, par le prisme du genre, sur ces territoires ressentis comme anxiogènes, sur ces lieux perçus comme excluants, et qui amputent la citoyenneté spatiale des minorités. Il convient alors d'entendre ici le rapport à l'espace mais également le rapport à la santé, non seulement comme l'absence de maladie, mais plus probablement comme l'état de bien être, dans lequel les questions de genre et de territoires ont toute leur place.

\section{BIBLIOGRAPHIE}

Alessandrin A. ; Espineira K., 2015, Sociologie de la transphobie, Bordeaux : MSHA. 
Alessandrin A., 2014, Folles in skies : éloge de la folle, Miroir / Miroirs vol.1, Des ailes sur un tracteur : pp 11-13.

Alessandrin A., 2014b, Transidentités : l'épreuve scolaire, Les cahiers de la transidentité, vol.3. Paris : Harmattan, pp : 39-50.

Alessandrin A., 2014c, Cisgenre, in Genre !, E-Bellebeau B., Alessandrin A. (dir.). Paris : des ailes sur un tracteur, pp : 29-31.

Alessandrin A., 2012, Le 'transsexualisme' : une nosographie obsolète. Revue française de santé publique, vol.24, $\mathrm{n}^{\circ} 3$, pp : 263-269. doi:10.3917/spub.123.0263

Alessandrin A., 2012b, Existe-t-il une culture trans, in La transyclopédie, Espineira K., Thomas MY., Alessandrin A. (dir.). Paris : des ailes sur un tracteur, pp : 124-125.

Amari S., 2013, Lesbiennes et migrantes dans la ville, in Géographie des homophobies, Alessandrin A. et Raibaud Y. (dir). Paris, Armand Colin, pp : 45-55.

Bastien-Charlebois J. ; Guillot V., 2013, Géographie du mouvement intersexe, in Géographie des homophobies, Alessandrin A. et Raibaud Y. (dir). Paris, Armand Colin, pp : 241-265.

Blidon M., 2013, Vers un observatoire régional de l'homophobie ?, in Géographie des homophobies, Alessandrin A. et Raibaud Y. (dir). Paris, Armand Colin, pp : 269-281.

Blidon M., 2006, La géographie est-elle queer ? Quand la question des identités sexuées et sexuelles ouvre de nouvelles perspectives... in Demain la géographie ? Permanences, dynamiques, mutations : Pourquoi ? Comment?, Groupe Dupont (dir). Avignon, France, pp.49-52.

Bochet B ; Racine J-B., 2002, Penser et connaître la ville, Géocarrefour, vol. 77, n ${ }^{\circ}$ 2, pp : 117-132.

Borghi R., 2014, L'espace à l'époque du queer : contaminations queer dans la géographie française, Politiqueer, $\mathrm{n}^{\circ} 1$ [en ligne]. Consulté le 10/03/2015. Disponible sur : http:// politiqueer.info/numeros/rpqfrancofolles/espace-queer/

Borghi R., 2012, De l'espace genré à l'espace « queerisé ». Quelques réflexions sur le concept de performance et sur son usage en géographie, ESO : travaux et documents, $\mathrm{n}^{\circ} 33$, pp : 109-115. Browne K. ; Lim J., 2010, Trans lives in the 'gay capital of the UK', Gender, Place and Culture, vol. $17, \mathrm{n}^{\circ} 5, \mathrm{pp}: 615-633$.

Browne K. ; Nash C.J. ; Hines S., 2010 Introduction: towards trans geographies, Gender, Place and Culture, vol.17, $\mathrm{n}^{\circ}$ 5, pp: 573-577.

Califia P., 2003, Le mouvement transgenre. Paris : Epel.

Cattan N., Leroy S., 2013, Atlas des sexualités, Paris : autrement.

Clerval A. ; Cattan N., 2011, Un droit à la ville : réseaux virtuels et centralités éphémères des lesbiennes dans Paris, Revue Justice spatiale n ${ }^{\circ} 3$ [en ligne]. Consulté le 19/07/2014. Disponible sur : http://www.jssj.org/article/un-droit-a-la-ville-reseaux-virtuels-et-centralites-ephemeresdes-lesbiennes-a-paris/

Doan P-L., 2010 The tyranny of gendered spaces - reflections from beyond the gender dichotomy, Gender, Place \& Culture: A Journal of Feminist Geography, vol.17, n ${ }^{\circ} 5$, pp: 635-654.

Doan P-L., 2007, Queers in the American City: Transgendered perceptions of urban space Gender, Place \& Culture: A Journal of Feminist Geography, vol.14, $\mathrm{n}^{\circ} 1, \mathrm{pp}:$ 57-74.

Ellis C., 2004, The Ethnographic I: A methodological novel about autoethnography. Walnut Creek : AltaMira Press. 
Espineira K., 2014, La médiatisation des politiques transgenres : du statut de contre-public à l'inégalité de la représentation, Revue française des sciences de l'information et de la communication $\mathrm{n}^{\circ} 4$ [En ligne], 4 |. Consulté le 28 mai 2014. Disponible sur : http:// rfsic.revues.org/695

Espineira K., 2013, Les trans studies face aux résistances académiques. Les cahiers de la transidentité. vol.2, pp : 9-16.

Espineira K., 2013, Transidentités et média(tion)s, in Géographie des homophobies, Alessandrin A. et Raibaud Y. (dir). Paris, Armand Colin, pp : 119-135.

Espineira K., Thomas M-Y, Alessandrin A., 2012, La transyclopédie : tout savoir sur les transidentités. Paris : des ailes sur un tracteur.

Espineira K., 2008, La transidentité : de l'espace médiatique à l'espace publique. Paris : Harmattan, coll. Champs visuels.

Foerster M., 2008, Histoire des transsexuels en France, H\&O, 2008.

Gutowski Y., 2013, Genre et géographie : La transphobie et son influence sur les pratiques des personnes transidentitaires dans l'espace public. Le cas de Paris et sa region . (Hancock C. dir), mémoire de master2, UPEC.

Halberstam J., 2005, In a queer time and place : transgender bodies, subcultural lives. NYU press. Hill D., 2002, Genderism, transphobia, and gender bashing : A framework for interpreting antitransgender violence, in Understanding and dealing with violence: A multicultural approach . (B. Wallace ; R. Carter dir.), Thousand Oaks. pp. 113-136.

Jaurand E., 2015, Territoires de mauvais genre : les plages gays, Revue Géographie et cultures n ${ }^{\circ}$ $54, \mathrm{pp}: 71-84$.

Le Talec J-Y, 2013, L'espace des folles, in Géographie des homophobies, Alessandrin A. et Raibaud Y. (dir). Paris, Armand Colin, pp : 225-240.

Leroy S., 2010, Bats-toi ma sœur : Appropriation de l'espace public urbain et contestation de la norme par les homosexuels, Métropoles $n{ }^{\circ} 8$ [En ligne], 8 | 2010. Consulté le 19 juillet 2014. URL : http://metropoles.revues.org/4367

Leroy S., 2009, La possibilité d'une ville : comprendre les spatialités homosexuelles en milieu urbain, Espaces et sociétés vol. $4 \mathrm{n}^{\circ}$ 139, pp : 159-174.

Leroy S., 2005, Le Paris gay : éléments pour une géographie de l'homosexualité, Annales de géographie vol.6 $\mathrm{n}^{\circ}$ 646, pp : 579-601.

Lestrade D., 2003, Exhibitionnisme, in Dictionnaire de l'homophobie, Tin, L.-G. (dir). Paris, PUF, pp. 160-162.

Moss P., 2001, Placing autobiography in geography. Syracuse University Press.

Namaste K., 1996, Genderbashing : sexuality, gender and the regulation of public space, Environment and Planning D : Society and Space. Vol.14, ${ }^{\circ}{ }^{\circ} 4$. pp. 221-240.

Outrans, 2013, Quelle visibilité pour les trans (entretien avec A. Alessandrin), in Géographie des homophobies, Alessandrin A. et Raibaud Y. (dir). Paris, Armand Colin, pp : 71-85.

Piat C., 1979, Elles... les travestis ; la vérité sur les transsexuels , Paris : Pocket.

Raibaud Y., 2013, Géographie de l'homophobie, Les cahiers de la transidentité vol.2, Harmattan, pp : 71-86. 
Serano J., 2007, Whipping girl : transsexual woman on sexism and the scapegoating of feminity. Seal ed.

Stryker S., Whittle S., 2006, Transgender studies reader, Routledge.

Thomas M-Y., 2013, L'exemple des UEEH, in Géographie des homophobies, Alessandrin A. et Raibaud Y. (dir). Paris, Armand Colin, pp : 119-135.

Thomas M-Y, 2012, UEEH, in La transyclopédie, Espineira K., Thomas M-Y., Alessandrin A. (dir.). Paris : des ailes sur un tracteur, pp : 307-309.

Zabus C., 2013, Transgender experience: place, ethnicity and visibility. Routledge.

\section{NOTES}

1. «Observatoire Bordelais de l'Egalité » (2014-2015)

2. Ce constat, d'une intégration intracommunautaire limitée, avait déjà été travaillé par Charlotte Prieur dans un texte intitulé "L'homophobie: une discrimination parmi d'autres dans les milieux LGBTQ» et disponible sur: http://www.observatoire-destransidentites.com/page-7779410.html

3. Je remercie Claire Hancock pour m'avoir indiqué certains de ces travaux

4. Lire l'entièreté du rapport sur: http://liverpool.gov.uk/media/100661/Transpeople\%27s-experiences-in-Liverpool-LCVS-Nov-2011.pdf

5. Lire à ce sujet la revue de presse de l'ODT (Observatoire Des Transidentités) d'octobre 2013 autour de l'existrans. Disponible sur: http://www.observatoire-destransidentites.com/article-existrans-revue-de-presse-120842289.html .

6. A cet égard, se référer au séminaire "Géographie des émotions ", sous la direction de Pauline Guinard et Bénédicte Tratnjek. Site: http://www.geographie.ens.fr/Geographie-des-emotions-.html

7. Le real life test est une étape du protocole de changement de sexe qui consiste à faire la preuve de la persistance de la demande en vivant et en éprouvant le genre ressenti. En tant qu'il est une injonction psychiatrique et qu'il est recommandé parfois sans hormonothérapie, ce real life test est très critiqué. Lire par exemple: Arnaud Alessandrin, "Santé psychiatrique: le principe de précaution est-il un principe éthique? » Ethique et Santé, vol.11, $\mathrm{n}{ }^{\circ} 1, \mathrm{pp}:$ 43-50, 2014.

8. L'enquête est consultable sur : http://chrysalidelyon.free.fr/sondage_sante2011.php

9. « Nous n'irons plus au bois », Josée Dayan, 2007.

10. «Screaming queens », Susan Stryker, 2005.

11. «Transamerica », Duncan Tucker, 2004.

12. « Laurence anyways », Xavier Dolan, 2012. 


\section{RÉSUMÉS}

À l'intersection des questions de genre, d'espace et de santé, se trouvent les questions transidentitaires. Cet article se propose de faire un premier état des lieux de la recherche géographique sur les questions trans, à partir des publications françaises et américaines, afin de souligner la nécessité de penser les espaces des transitions comme des lieux d'injonctions à la cisidentité, c'est-à-dire des lieux où règne le respect des assignations de genre. Il en découle de nombreuses tensions, relatives à l'expression de l'identité de genre des personnes trans mais également à leur santé ou à leurs représentations sociales et médiatiques. De ce point de vue, cet article se présente comme l'esquisse d'une géographie sociale des transidentités et participe à l'implémentation progressive des trans studies dans le paysage universitaire français.

At the intersection of gender, space, and health issues, are 'trans studies'. For a long time, the question of trans-identities have been contained in spaces which are, at the same time, on stage (the cabaret, the night's world), coded obscene (prostitution, physical transformations) or related to psychiatrisation, pathologisation... The world has limited its spaces of interactions with trans people into what are often 'suffocated' spheres: the surgery block and some disgracing press coverage. With the trans movement, as a structured and politicized social movement, the visibility of transidentities has increased. Trans associations, as well as the news media, have established a new polity of visibility, that is more complex and less pathologized. The geography of transidentities gradually leaves the secret of doctor's offices to conquer more supports (on screens), legal and public interfaces. In France nevertheless, research around the spaces of transidentities is still limited. Trans studies, themselves are new in the French academic field (Espineira, 2008) and sometimes have difficulty being heard (Espineira, 2013). As such, the attempts to create LGBT geographies remain mainly focused on a "gays" analysis (Jaurand, 2005; Leroy, 2005, 2009; Raibaud, 2013) or "lesbian" research (Clerval and Cattan, 2011; Amari, 2013). International litterature is, more plentiful (Doan, 2007). Now, trans courses disrupts the supposed neutrality of space and highlight its stressful size, in particular when the gender expression does not return to sex assignment at birth.... The trans testimonies in particular, insist frequently on the spaces of intimity which are rare and precious and submitted to multiple controls by the parents, by relatives or by doctors, bruising privacy. At another scale, spaces of health and media spaces are good markers of the tensions which mark out the trans lifecourses. With consideration given to this context, the first part of this article examines the literature dealing with the space and the transidentities. Then in a second part the purpose is to examine the way the trans people are involved in more or less stressful spaces, more or less visible spaces also, and the way women's and homosexual movements helped them to build an interface for dissident gender expression. It will be necessary, also, to underline that the geography of transidentites creates favorable maps to analyse the neutral or impersonal reading of the space and inaugurates a particular reading of the space and the interactions which take place there. A geography of transidentities shows, in hollow, a geography of the "cisgender privilege" to resume the expression of Julia Serano (2007). From this point of view, this article appears as a sketch for one social geography of transidentities and the progressive implementation of trans studies in the French university landscape.

\section{INDEX}

Keywords : transidentity, gender, geography, health, spaces 


\section{AUTEUR}

\section{ARNAUD ALESSANDRIN}

Docteur en sociologue, chercheur associé au centre Emile Durkheim, Université de Bordeaux).

Co-Fondateur de l'O.D.T. (Observatoire Des Transidentités). Site : http://www.observatoire-destransidentites.com . 